\title{
Article \\ The Muscle Fatigue's Effects on the sEMG-Based Gait Phase Classification: An Experimental Study and a Novel Training Strategy
}

\author{
Jianfei Zhu ${ }^{1,+}{ }^{\oplus}$, Chunzhi Yi ${ }^{1,2,+} \mathbb{C}$, Baichun Wei ${ }^{2}$, Chifu Yang ${ }^{1}$, Zhen Ding ${ }^{1}$ and Feng Jiang ${ }^{2, *}$ \\ 1 School of Mechatronics Engineering, Harbin Institute of Technology, Harbin 150001, China; \\ nyzhujianfei@163.com (J.Z.); chunzhiyi@hit.edu.cn (C.Y.); cfyang@hit.edu.cn (C.Y.); \\ 16b908060@stu.hit.edu.cn (Z.D.) \\ 2 School of Computer Science and Technology, Harbin Institute of Technology, Harbin 150001, China; \\ 18B903018@stu.hit.edu.cn \\ * Correspondence: fjiang@hit.edu.cn \\ + These authors share the same contribution to the study.
}

Citation: Zhu, J.; Yi, C.; Wei, B.; Yang, C.; Ding, Z.; Jiang, F. The Muscle Fatigue's Effects on the sEMG-Based Gait Phase Classification: An

Experimental Study and a Novel Training Strategy. Appl. Sci. 2021, 11, 3821. https://doi.org/10.3390/ app11093821

Received: 28 February 2021

Accepted: 21 April 2021

Published: 23 April 2021

Publisher's Note: MDPI stays neutral with regard to jurisdictional claims in published maps and institutional affiliations.

Copyright: (c) 2021 by the authors. Licensee MDPI, Basel, Switzerland. This article is an open access article distributed under the terms and conditions of the Creative Commons Attribution (CC BY) license (https:// creativecommons.org/licenses/by/ $4.0 /)$.

\begin{abstract}
Surface Electromyography (sEMG) enables an intuitive control of wearable robots. The muscle fatigue-induced changes of sEMG signals might limit the long-term usage of the sEMG-based control algorithms. This paper presents the performance deterioration of sEMG-based gait phase classifiers, explains the deterioration by analyzing the time-varying changes of the extracted features, and proposes a training strategy that can improve the classifiers' robustness against muscle fatigue. In particular, we first select some features that are commonly used in fatigue-related studies and use them to classify gait phases under muscle fatigue. Then, we analyze the time-varying characteristics of extracted features, with the aim of explaining the performance of the classifiers. Finally, we propose a training strategy that effectively improves the robustness against muscle fatigue, which contributes to an easy-to-use method. Ten subjects performing prolonged walking are recruited. Our study contributes to a novel perspective of designing gait phase classifiers under muscle fatigue.
\end{abstract}

Keywords: electromyography; gait phase classification; muscle fatigue; wearable robots

\section{Introduction}

Lower-limb wearable robots, a kind of powered device that can enhance the mobility of the human body, have been widely used for assistive and rehabilitative purposes in recent years [1-3]. Regarding the control of wearable robots, surface Electromyography (sEMG) provides an intuitive signal source that can directly reflect users' motion intent, and thus, can assist in involving the active participation of users [4-7]. Among the applications of sEMG signals in lower-limb wearable robots, gait phase classification provides the fundamental information of lower-limb movements, thus can form effective assistance and can be used in disease diagnosis [8,9]. Much research that classified gait phases used multiple features and classifiers with accuracy over $90 \%$, which are of practical value [10-12]. However, as indicated in the study [13], the nonstationary characteristics of sEMG signals that can be attributed to many extrinsic or intrinsic factors might limit the broader applications of sEMG. Among all the factors that might affect the performance of the sEMG-based classifiers, muscle fatigue is the intrinsic factor that seems to be an unavoidable factor, especially during the long-term usage of wearable robots.

Fatigue is defined as the decrease in muscle contractility after prolonged excises, usually measured through the muscle force deterioration during the maximum voluntary contractions. Many studies have reported some basic findings in the domain of SEMG and fatigue. One conclusion is that muscle fatigue will induce the dynamic range increase and the frequency decrease of the sEMG signals [13-15]. Based on this phenomenon, a lot of features, such as root mean square (RMS) [16,17], median frequency (MDF) [17,18], mean 
power frequency (MPF) [18,19], and wavelet transform (WT) [19,20], have been studied to indicate the development of muscle fatigue, and research suggests their availabilities as the indices of fatigue. These studies usually plotted the values of the employed features in the course of time, without considering how the changes of the values affect the performance of classifiers.

Regarding the fatigue-induced change of the sEMG-based classifiers' performance, several studies have been performed. Tkach et al. [21] studied the influence of three factors, including muscle fatigue, on the classification of four contraction patterns, which focused on time-domain features. Bukhari et al. [22] performed the experiments to investigate the performance of the K-nearest neighbor $(\mathrm{KNN})$ classifier, which was used to classify contraction patterns of biceps brachii under muscle fatigue. Although presenting impressive inspirations, how the non-fatigue data-trained classifiers would deteriorate on fatigue data might still be a research gap. Moreover, as for the gait phase classification, how muscle fatigue affects the performance of the gait phase classification is still unclear.

Pioneer studies also focused on improving the classifiers' robustness against muscle fatigue. Song et al. [23] and Ding et al. [24] have proposed EMG based gesture classifier, which is robust to fatigue by using a fuzzy neural network and an adaptive incremental hybrid classifier-based recognize strategy, respectively. Mainardi et al. [25] developed a new double differential electrode that can compensate for the influence of muscle fatigue by modifying its gain. Those efforts are directed to measuring the impact of fatigue on locomotion mode classification and attempt to build anti-fatigue classifiers. However, these studies either require a relatively large computation, due to the employed complex methods or require specially designed equipment. These might deviate from the easy-touse demand, thus might limit the broader applications. In our study, we propose a training strategy to improve the classifiers' robustness against muscle fatigue, which could be a simpler way.

In our work, two basic classifiers are tested on real sEMG data collected from prolonged walking to investigate the influence of muscle fatigue. By dividing the data in chronological order, we get non-fatigue sets and fatigue sets. We train the gait phase classifiers by the non-fatigue set and test them by the fatigue set to reveal their performance under fatigue. In addition, different features are used in the classification to compare their performance. In order to solve the deterioration of the classifiers, we propose a training strategy that adds part of the fatigue set into a training set, which presents a promising improvement. Ultimately, we perform the analysis of variance (ANOVA) on the feature extracted from the sEMG signals to interpret the phenomena in the classification. Figure 1 shows how this research is conducted. Our contributions can be summarized, as follows.

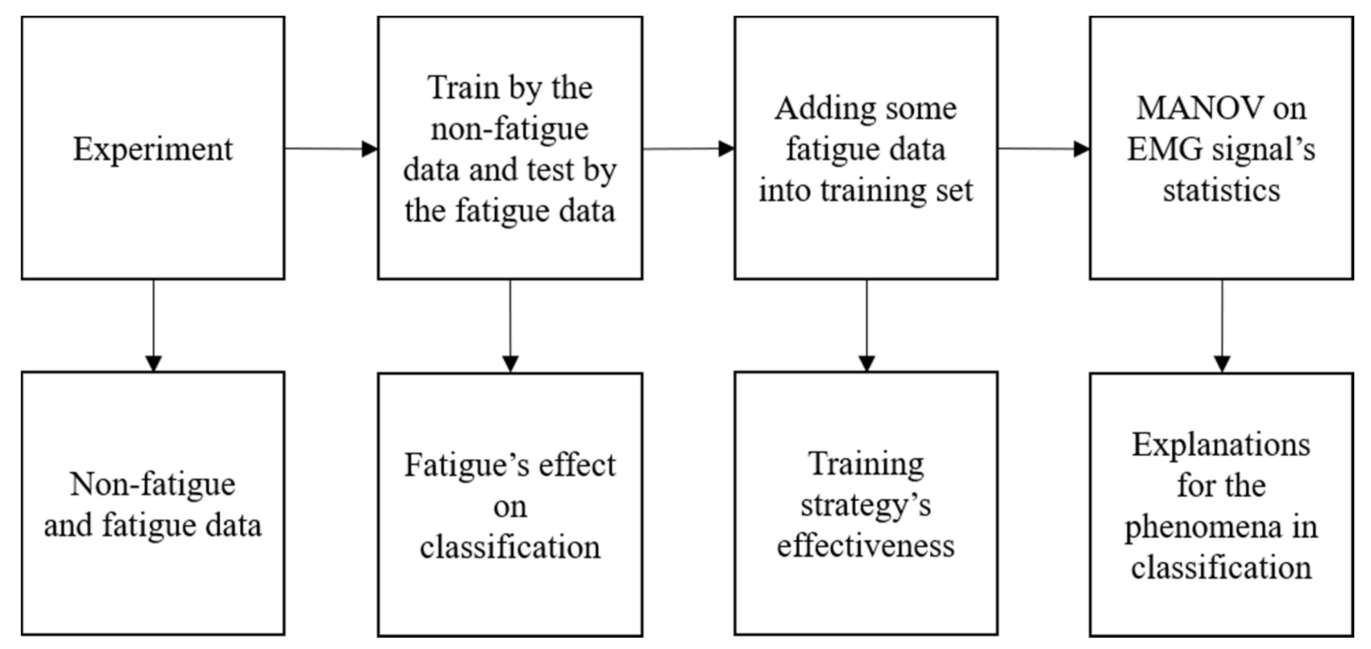

Figure 1. Flowchart of our research. 
- To the best of our knowledge, this work is the first study that shows how the gait phase classifiers are affected by muscle fatigue;

- We propose a training strategy to improve the classifiers' robustness against muscle fatigue;

- We explain the phenomena of the classification by quantitatively analyzing the timevarying characteristics of sEMG features under muscle fatigue.

\section{Related Work}

Before the statement of our method, it is necessary to introduce some previous research, which provides some inspirations.

In order to perform our study, we have to design an appropriate experiment to induce muscle fatigue. The experiment protocols to induce lower-limb muscle fatigue are mainly divided into the following three kinds: Isokinetic contraction, repetitive sit-to-stand, and endurance task.

The isokinetic contraction or the repetitive sit-to-stand may not be an appropriate way to induce muscle fatigue-related change in gait. Granacher et al. [26] induced the local muscle fatigue by the isokinetic knee extension, and they studied the impact of the fatigue on human gait. Their results showed that the local muscle fatigue induced by the isokinetic contraction only evoked a subtle change in human gait even if the decrease of the maximum voluntary contraction (MVC) reached 50\%. Barbieri et al. [27] reported similar results. In their study, the subjects were asked to perform the sit-to-stand until they were exhausted. The results also showed that the MVC of the subjects decreased by $12 \%$ on average, but the gait parameters only changed diminutively. Morrison et al. [28] studied the fatigue induced by endurance tasks on a treadmill, and they tested the gait by walking overground. According to their results, obvious gait changes were detected.

The results of the above studies indicate that the way to induce muscle fatigue may count, and it is better to select an endurance task similar to the test motion. The conclusion seems to be the common practice for the sEMG-related muscle fatigue studies, such as the studies that induced fatigue by running on a treadmill [29,30]/overground [31], walking [32], and cycling [33-35]. In this paper, we study the effect of muscle fatigue in walking, so long walking time on a treadmill was selected as the method to induce fatigue.

In our study, muscle fatigue will be investigated through the observation of the classification accuracy, so it is also necessary to appropriately select the features used in the classification. Our intent is not only to cover the features commonly used in the classification, but also to involve the features that can be used in fatigue detection.

Many features have been used as indices to measure muscle fatigue in previous research. With the deepening of muscle fatigue, the sEMG signal varies according to a trend of dynamic range increase and frequency decrease [13-15,36]. Based on this phenomenon, many studies have reported the abilities of the traditional EMG features to detect muscle fatigue. Root mean square (RMS) was used to detect the amplitude changes [16,37], while median frequency (MDF) [18,37] and mean power frequency (MPF) $[18,38]$ were used to detect the frequency changes. In this paper, these three features are selected.

Many studies have reported the effect of muscle fatigue on EMG signals, and some of them also analyze it from the aspect of classification accuracy. Although these works mainly focused on upper limbs, it is necessary to introduce their studies as references to our work.

Tkach et al. [21] studied the stabilities of eleven features under muscle fatigue by testing the classifiers' accuracies. In their study, the subjects performed arm motions by a customized handle. The short-term muscle fatigue was induced by isometric contractions. The classifiers were used on types of contraction during arm motions, and they trained the classifiers by the non-fatigue data, while testing them by the fatigue data. Their results showed that muscle fatigue significantly affects only one of the eleven features from the perspective of the accuracy of the classifier. However, as mentioned above, using an isometric contraction to induce short-term muscle fatigue may not induce a similar muscle 
pattern change of long-term walking, thus might not affect the performance of classifiers in the same manner.

Bukhari et al. [22] have studied the performance of the K-Nearest Neighbors (KNN) classifier on the non-fatigue data and the fatigue data. In their study, the subjects were asked to perform the "bicep curl", and muscle fatigue was induced by the repetitive motion. They also use the classifiers to identify the contraction type of the biceps brachii. The classifiers were implied separately on the non-fatigue data and the fatigue data, and their results showed that the KNN classifier performed better on the non-fatigue data. This indicates that muscle fatigue may change the distribution of sEMG data, which impacts the effectiveness of the KNN classifier.

\section{Materials and Methods}

This section states the main procedures of our proposed method, which can be seen in Figure 2. This paper aims to investigate the performance of the gait phase classification under fatigue. As mentioned in Section 2, we chose a long walking time as the experiment method to induce muscle fatigue. The collected data is divided in a chronologic order to get the non-fatigue data and the fatigue data. According to the research goal, eight features are selected and extracted from the sEMG signals. Moreover, we train classifiers using the non-fatigue data and test them using the fatigue data to reveal the effects of muscle fatigue on the classification. With the purpose of improving the performance of the classifier, we propose a novel training strategy that adds some of the fatigue data into the training set, and test the strategy using the rest fatigue data.

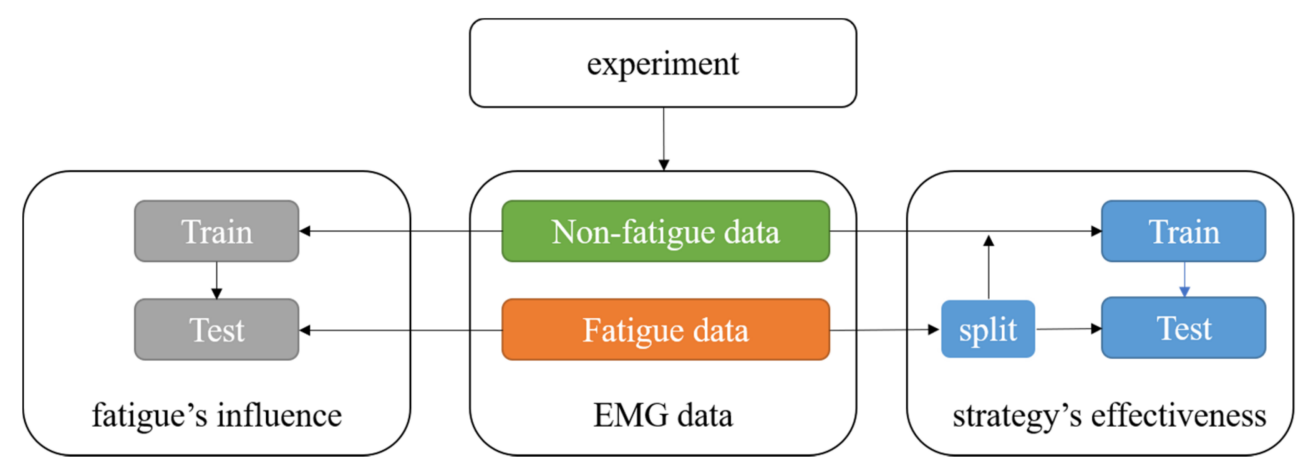

Figure 2. Main procedures of the proposed research.

\subsection{Experiment Protocol}

Ten healthy volunteers (five male and five female) participated in this study. None of them has ever suffered from any musculoskeletal or neuromuscular disease, and they were asked not to perform any intense physical exercise $48 \mathrm{~h}$ before the experiment. We explained the contents and the procedures of the experiment to the participants, ensuring that they were all aware of any possible risk. This study has got the permission of the local Ethics Committee.

The subjects were asked to walk on a treadmill for three sessions, each lasting $20 \mathrm{~min}$, without any rest at the intervals, and the speed of the treadmill was set constantly to $4.5 \mathrm{~km} / \mathrm{h}$. A speed of $4.5 \mathrm{~km} / \mathrm{h}$ was the normal speed of human walking, and walking for a total of $60 \mathrm{~min}$ can ensure that muscle fatigue was induced. While walking, sEMG was collected by the bipolar sensors (Trigno Wireless System; DELSYS, Boston, MA, USA) fixed on the skin of the right leg according to the positions of target muscles. The sensor positions were shown in Figure 3, and the following nine target muscles were selected: Rectus femoris (RF), vastus lateralis $(\mathrm{VL})$, vastus medialis $(\mathrm{VM})$, tibialis anterior (TA), soleus (SOL), semitendinosus (ST), biceps femoris (long head, BF), gastrocnemius lateralis (LG), gastrocnemius medialis (MG). Pressure sensors were placed at heels to get the information of ground reaction force, which were used to get the labels of the collected EMG data. In this study, a complete gait cycle of walking was divided into four phases, including 
loading response, mid-stance, terminal stance, and swing. Figure 4 showed a segment of the collected sEMG signal.

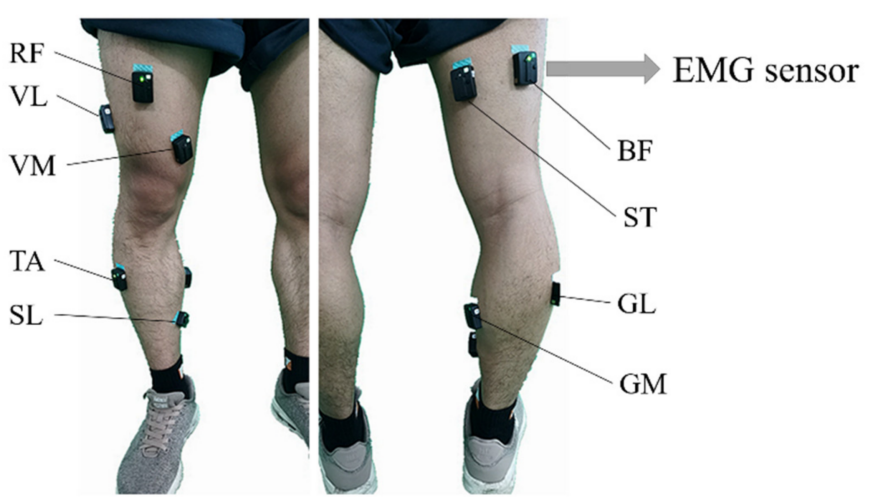

Figure 3. The positions of the sEMG sensors.

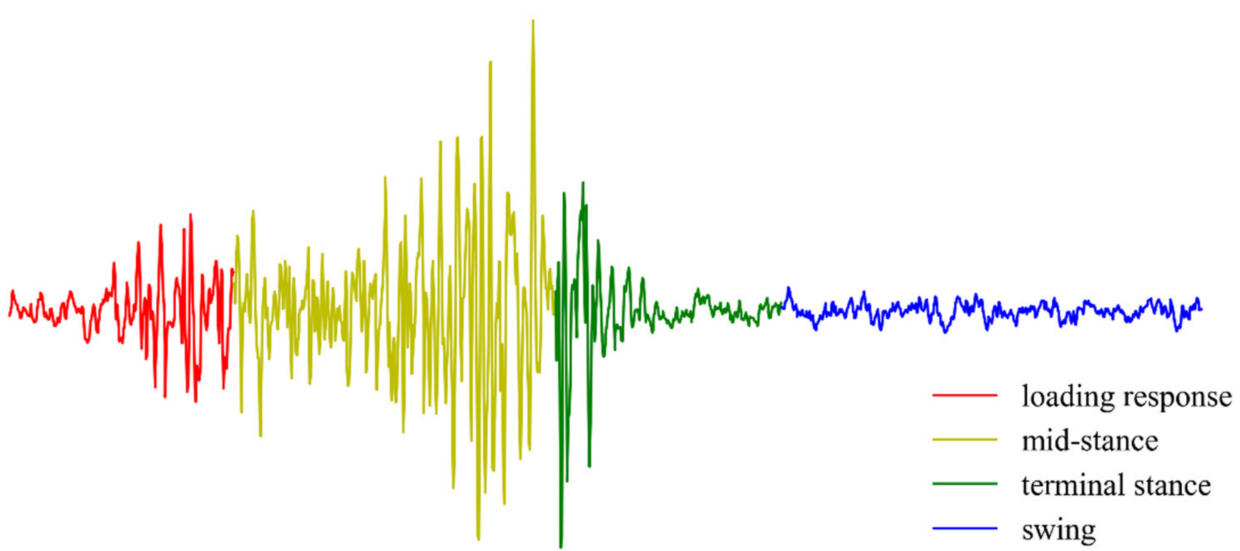

Figure 4. A sample segment of the raw sEMG signal. The color of the curve denotes the label (gait phases) of the signal.

\subsection{Selection of Features}

Selecting appropriate features is critical for the sEMG-based gait phase classification. The features can be divided into three categories: Time-domain (TD), frequency domain (FD), and time-frequency domain (TFD).

The TD feature can be obtained directly from the raw sEMG, which results in a small computation overhead and guarantees the real-time performance of the classifier [39]. In this study, we choose five TD features, including Hudgin's set (mean absolute value (MAV), zero crossings (ZC), waveform length (WL), and slope sign changes (SSC)), and RMS. Hudgin's set is the most widely used feature set in the sEMG- based pattern recognition with fair performance [40]. RMS is chosen because it can characterize the change of the sEMG amplitude caused by muscle fatigue [15].

The FD feature is extracted from the Fourier transform of the signal. In the FD features, we choose MDF and MPF because of their abilities to reflect the fatigue-caused frequency changes $[18,37,38]$. Although the TFD features also perform well in fatigue evaluation $[19,20]$, their practical application is still limited by the computational complexity. In this paper, the TFD features are not included.

In addition, a newly proposed feature spinal module action (SMA) is included in this study, which carries deeper physiological information in a "population coding" way to express movement.

In a word, eight features are considered in this paper, and they are MAV, ZC, WL, SSC, RMS, MDF, MPF, and SMA. 


\subsection{Data Process}

For the seven classic features, we select 128 -ms windows with 119-ms overlap to segment the sEMG signals. The TD features are calculated directly from the windowed signals, while the FD features are calculated from the Fourier transform of the signals. The calculation formulas of these features can be found in [41].

Before the extraction of SMA, we process the raw EMG to get its envelope as the following three steps: (1) $20-500 \mathrm{~Hz}$ Butterworth bandpass filtration, (2) calculation of the signals' absolute values, and (3) $10 \mathrm{~Hz}$ Butterworth lowpass filtration. The envelope is segmented by 1-s windows with 30-ms intervals, and the labels are decided by the windows' middle points. Each signal segment is decomposed into four dimensions by the Nonnegative Matrix Factorization. For each dimension of the segment, we calculate a threshold by adding the mean value and 1.5 times the variance. The four dimensions are binarized separately by their thresholds, where the values greater (less) than the according to the threshold are set to $1(0)$. After that, we pool the four binary sequences together by the bitwise operation. The pooled sequence is equally divided into ten subsequences, and the subsequences are summed separately to get the ten values. The ten-element vector is exactly the feature SMA of the window.

\subsection{Gait Phase Classification}

In this study, we select the support vector machine classifier (SVM) with Gaussian kernel and the decision tree classifier (DTC) to evaluate the impact of fatigue and the effectiveness of the training strategy. SVM can project data into the high-dimensional space in different forms depending on the choice of the kernel function to generate the classification boundaries, and it has been wildly used in the field of sEMG recognition with proved performance [42]. DTC is chosen, due to the small account of calculation, the easiness of understanding, and the extensive utilization in the classification of the biomedical signals [43].

As the experiment progressing, the muscle fatigue of the subjects continuously deepens. In order to investigate the relationship between the classifiers' performance and muscle fatigue, we consider that the subjects are not fatigued in session 1 and fatigued in session 3.

The classifications in this study are divided into two parts. (1) We chose the data in session 1 as the training set, while the data in session 3 as the test set. In this way, we try to determine how much muscle fatigue affects the gait classifiers and which classifier performs better under the fatigue. (2) The first half of the data of session 3 is added into the training set, and the rest of the data is used as the test set. In this way, we propose the training strategy that aims to improve the performances of the classifiers under the fatigue condition. Figure 5 shows the split of the data set in the classification.

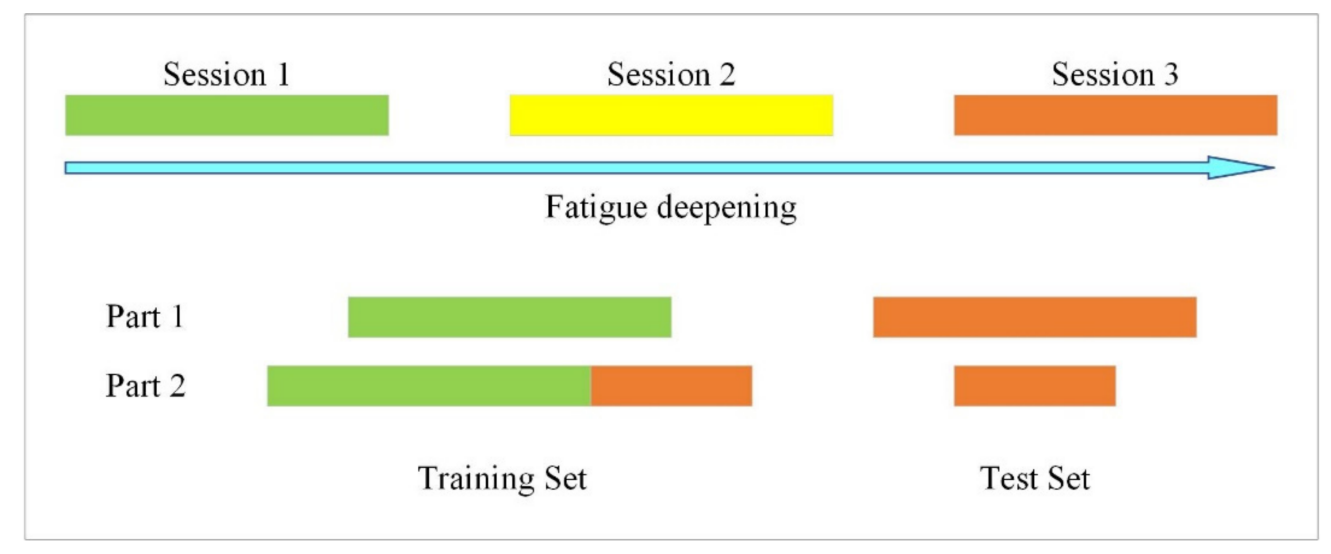

Figure 5. Split of the data set in the classification. 
In each part, we classify the gait phases by SVM and DTC with the eight features, respectively. Optimizations of the hyperparameters are carried out by the 5-fold crossvalidation in the training set, and then the models with optimized hyperparameters are trained by the training set and tested by the test set. The accuracies of the cross-validation and of testing on the test set are recorded. These procedures are performed in each subject separately, and the results are averaged over all the subjects.

\subsection{Results and Findings}

Results of part 1 are shown in Figure 6a, which reflects the performance of the classifier and how much muscle fatigue can affect the classifiers. It can be seen that the classifiers with different features have different performances. MAV, RMS, WL, and SMA have better performances than ZC, WL, SSC, MDF, and MPF. The classification deterioration caused by muscle fatigue can be reflected by the accuracy comparison between the train and the test. The accuracies become significantly lower for the six features, including MAV, RMS, ZC, SSC, MDF, and MPF, while the changes are not significant for WL and SMA. Among all of the eight features, SMA has the best performance under the muscle fatigue condition.

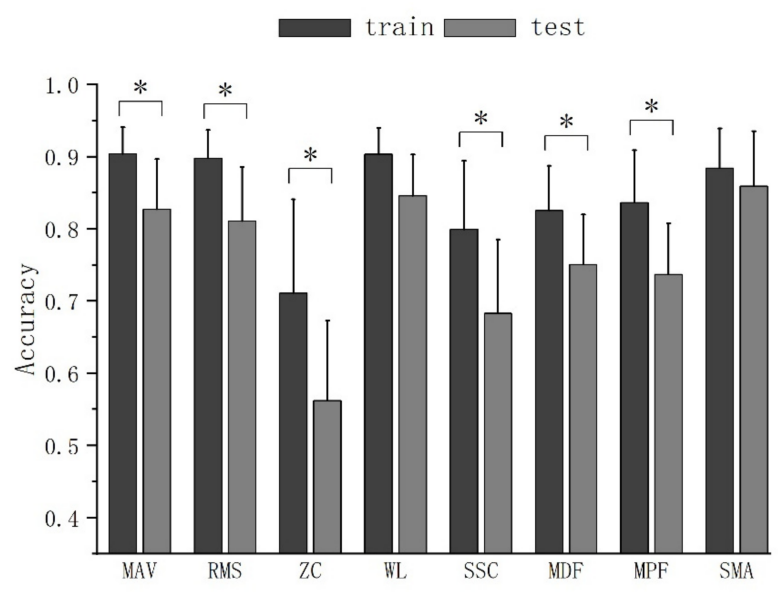

(a)

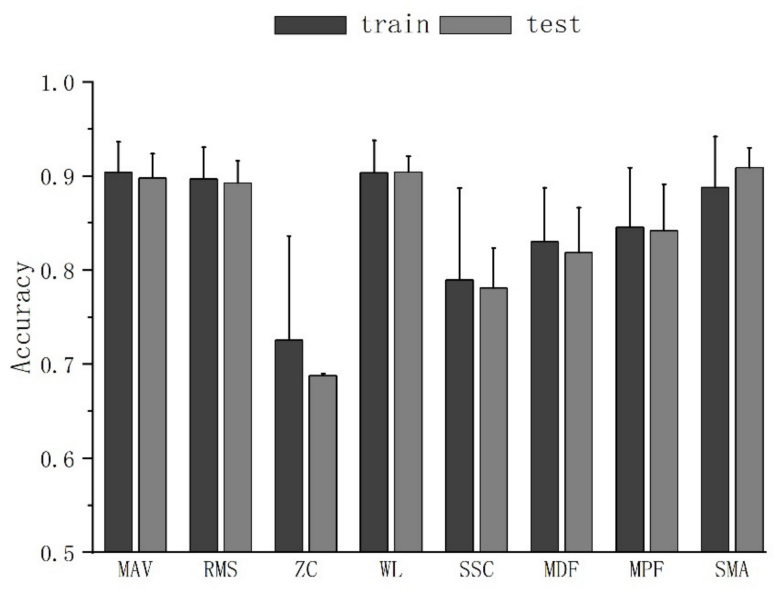

(b)

Figure 6. Results of the classification. (a) Results of part 1 that uses the non-fatigue data as the training set and the fatigue data as the test set; (b) results of part 2 that adds half of the fatigue data into the training set. The bars denote the mean value of accuracies, and the error bars denote standard deviation. Stars $\left(^{*}\right)$ indicate that the accuracies of the training and the test are significantly different by one-way ANOVA, and the significant level is set to $\alpha=0.05$. 
Figure $6 \mathrm{~b}$ shows the results of part 2 , which is to validate our training strategy. In this part, the accuracies do not significantly decrease when testing on the test set, and the classifiers even perform better on the test set than on the training set (i.e., the crossvalidation) in some cases. The accuracies of the cross-validation are not obviously lower than those in part 1 . These results suggest that our strategy improves the performance of the classifier under fatigue. Figure 7 displays a scenario of potential application.

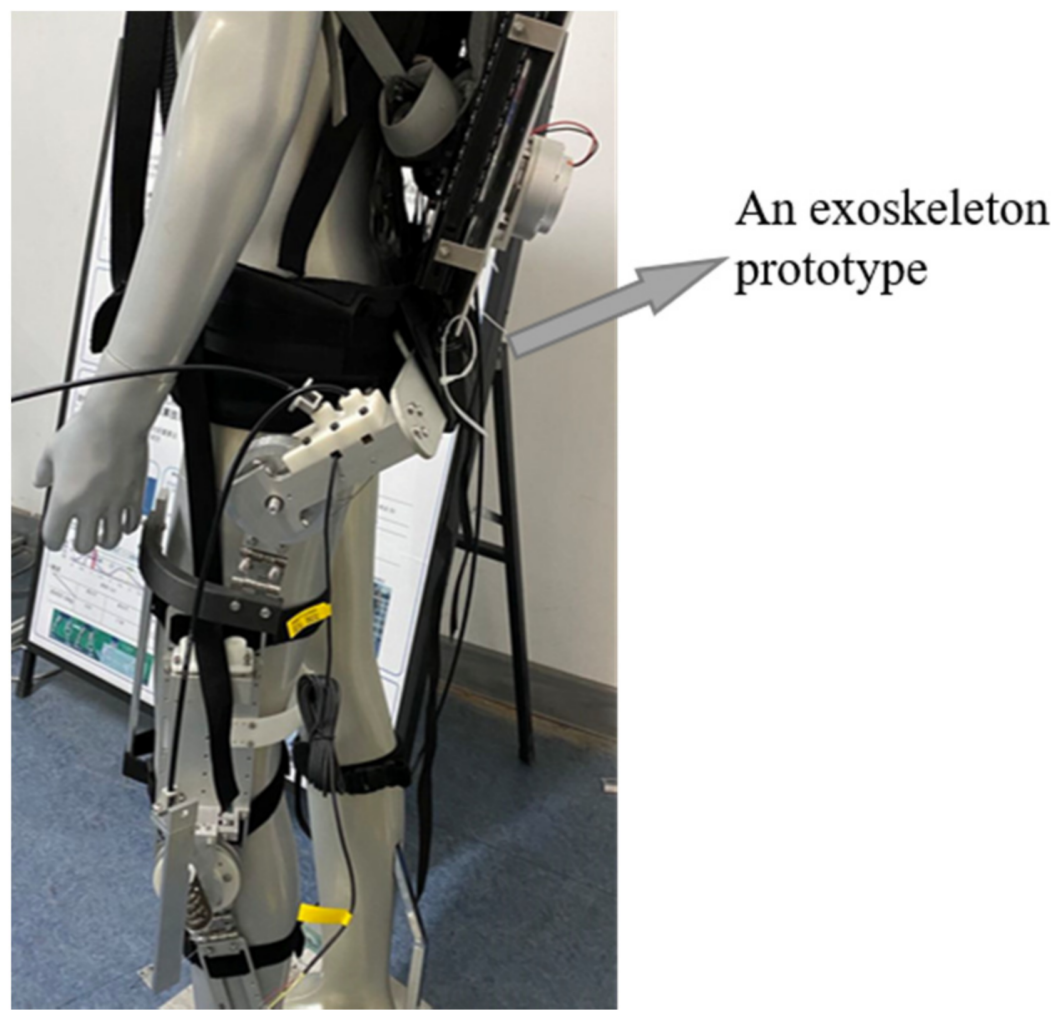

Figure 7. A potential application of our method.

\section{Analysis}

As mentioned above, the classification results reveal the effects of muscle fatigue on the different features and the effectiveness of our training strategy. But the deep reasons for these phenomena are still unclear, since these results are based on the classification accuracy. It is thus necessary to study the correlation between the EMG features and muscle fatigue from the perspective of statistical analysis. In this section, we directly investigate the extracted features using the Analysis of Variance (ANOVA) to validate whether and how much muscle fatigue affects the EMG features. We expect to explain the phenomena of classifiers through the results of the analysis.

The ANOVA is a statistical method that interprets the total variation of the data as the sum of the intra-group and inter-group variation in each level, and uses the F-test to determine whether the factors have significant effects on the dependent variable. The data to be analyzed in this section satisfies the conditions of ANOVA that independently sampling and similar variance.

Before the statistical analysis, the sEMG signals need to be processed. We split the signal into 4-s segments, and calculate the mean and the variance for each segment. The segment length is set to $4 \mathrm{~s}$ that approximately covers five gait cycles to denote the characteristics of EMG signals at the current instance. We assume the fatigue would not deepen in the 4-s duration. In the analysis, the degree of muscle fatigue is described more accurately that every 2 min containing 30 segments is considered as a fatigue level.

We adopt the Multivariate Analysis of variance (MANOVA) to analyze the features from multiple channels. In addition, other than muscle fatigue, the personalized charac- 
teristics of the sEMG signals also significantly affect the performance of the classifiers. In order to exclude the confounding factor, we perform the two-way MANOVA. We use the Principal Components Analysis (PCA) to reduce the dimension of SMA, remaining one principal component, and the rest treatments are the same as the seven rest features. In this section, the significant level is set to $\alpha=0.05$.

\section{Results}

The trends of the mean values of the sEMG features are shown in Figure 8. For the seven features, including MAV, ZC, WL, SSC, RMS, MDF, MPF, the results reveal that muscle fatigue significantly affects their values ( $p<0.05$ both for mean and variance). It can also be seen in Table 1 that the degree of the correlation between the seven features and the individuals is greater than that of the fatigue. As for SMA, that the significance level of the mean is $p=0.029$ for muscle fatigue and $p=0.996$ for the individuals. That is to say, the local average levels of SMA have significant differences under the different fatigue levels, but do not have significant differences under the different individuals. For the variance, the significance levels are $p<0.05$ for both muscle fatigue and the individuals, which indicates the significant differences. Table 1 shows the $F$ values of the features. Compared with the seven rest features, SMA has a much smaller $F$ value of mean for muscle fatigue which indicates that the local average levels of SMA have a weaker relationship to muscle fatigue.

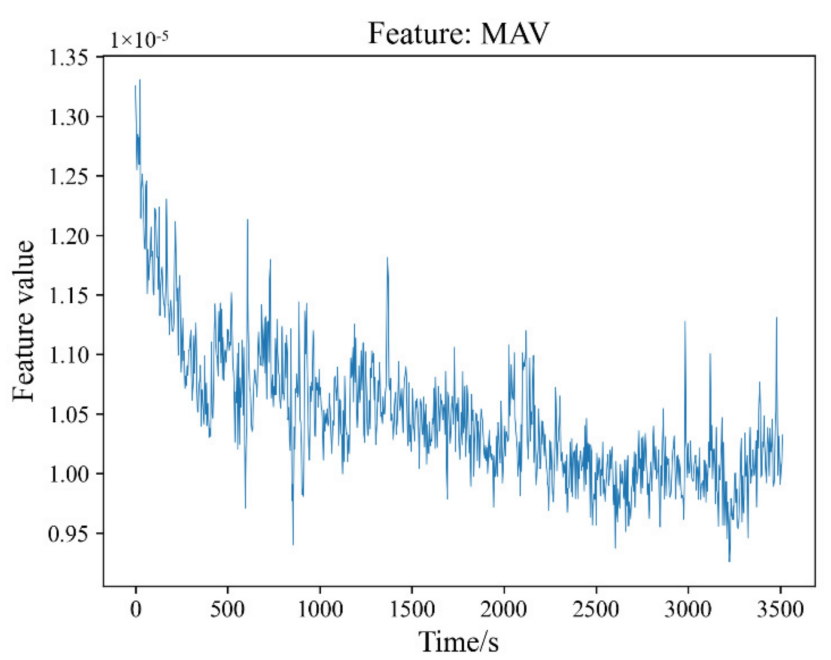

(a)

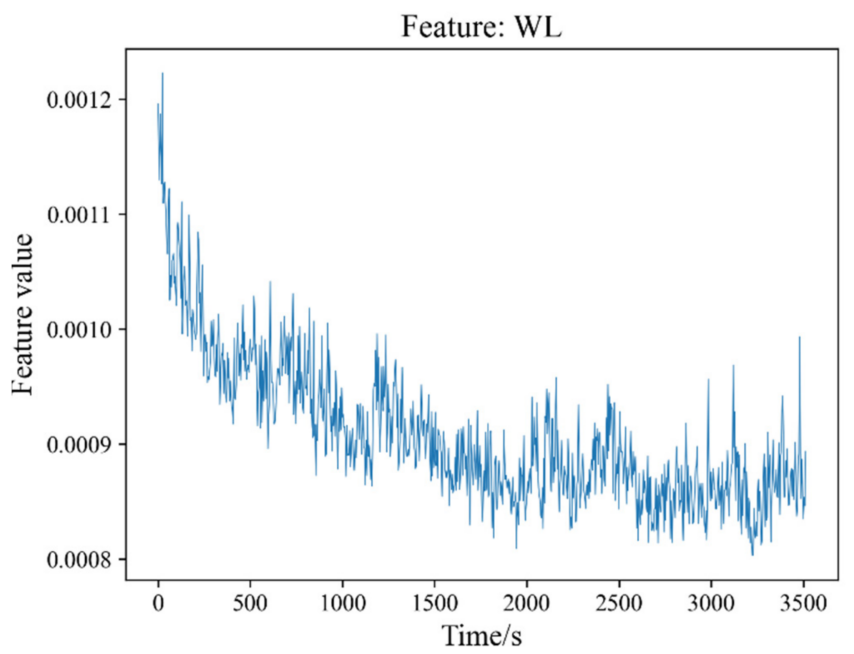

(c)

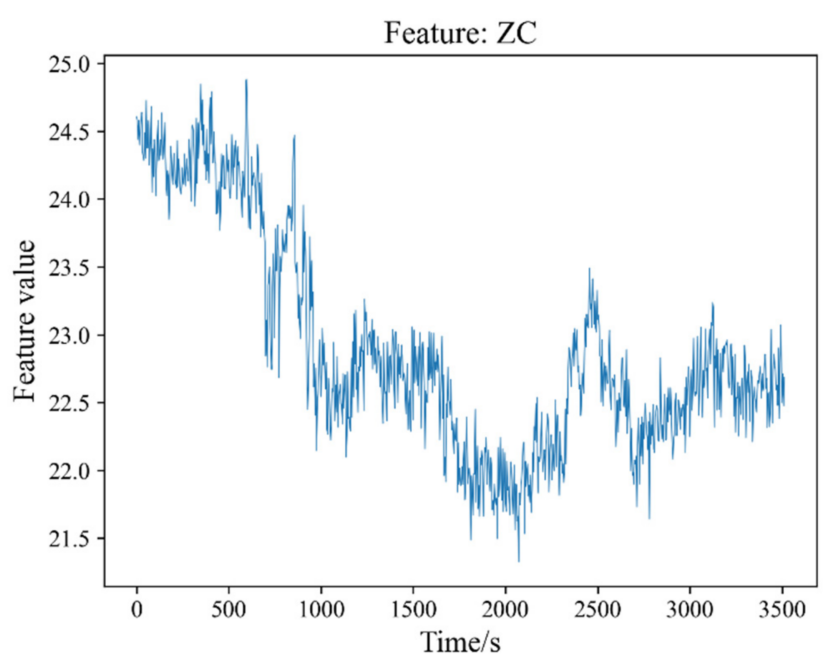

(b)

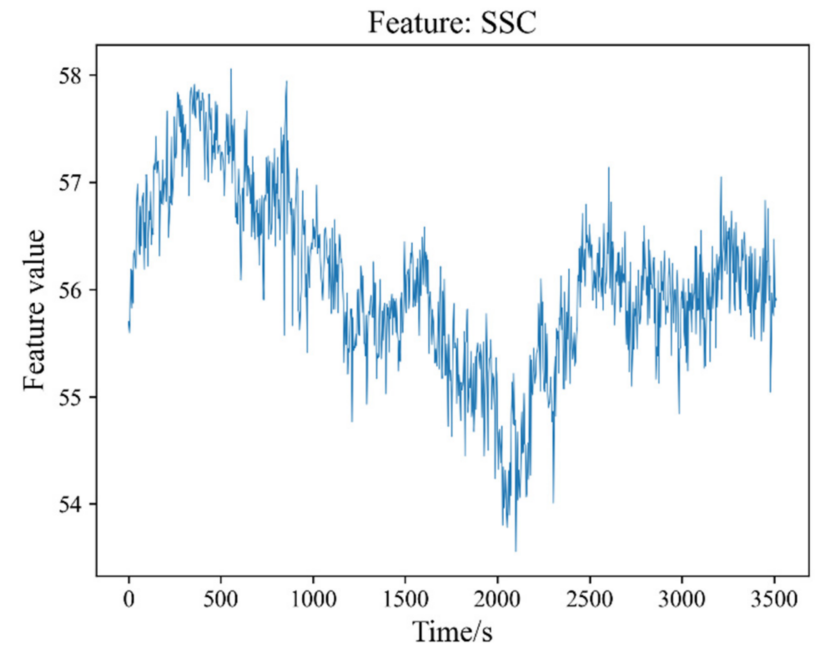

(d)

Figure 8. Cont. 


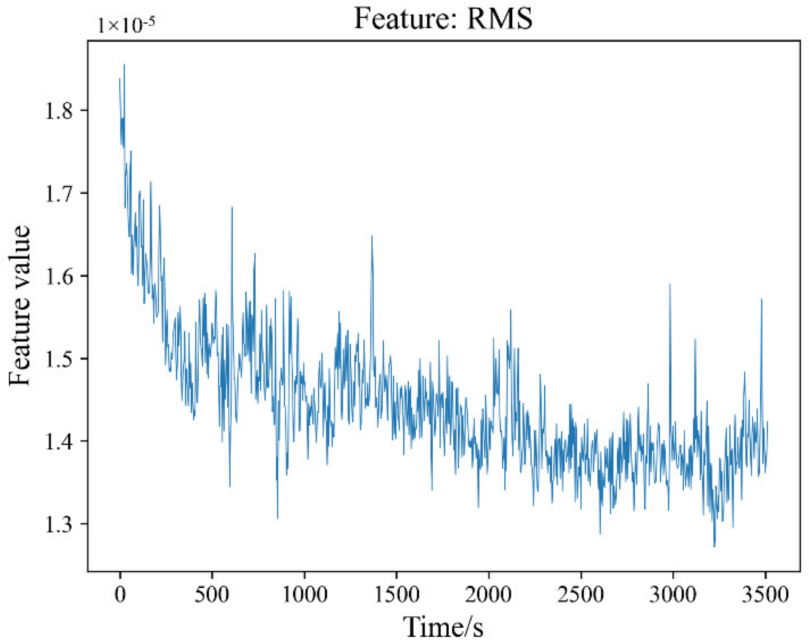

(e)

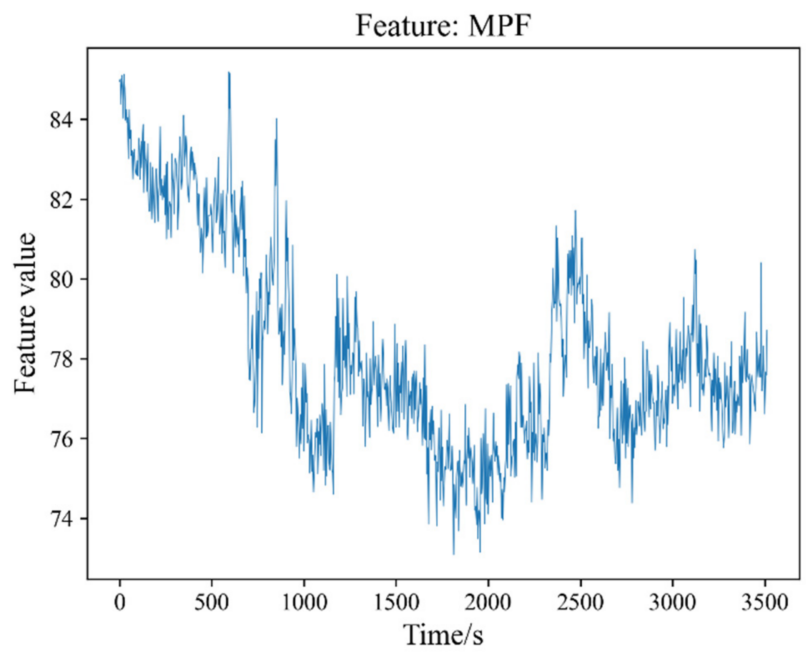

(g)

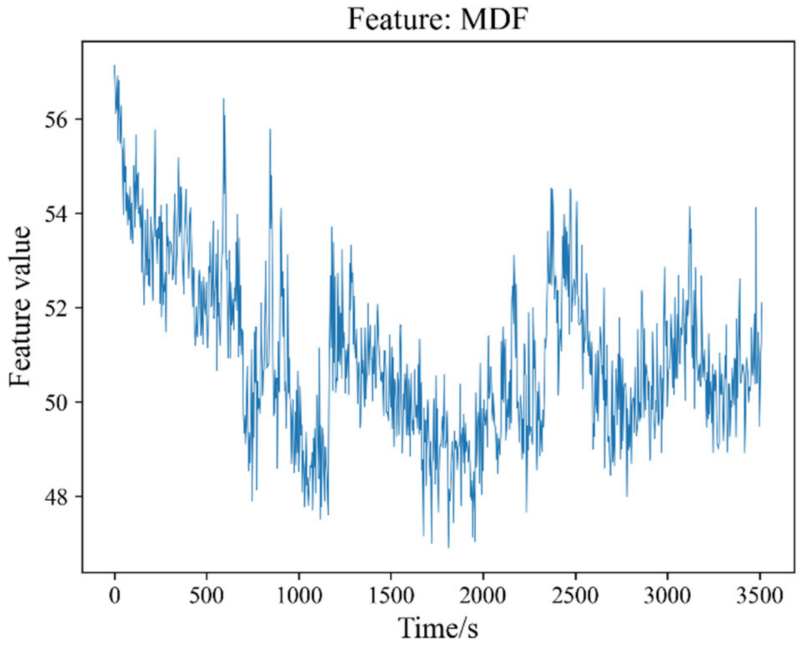

(f)

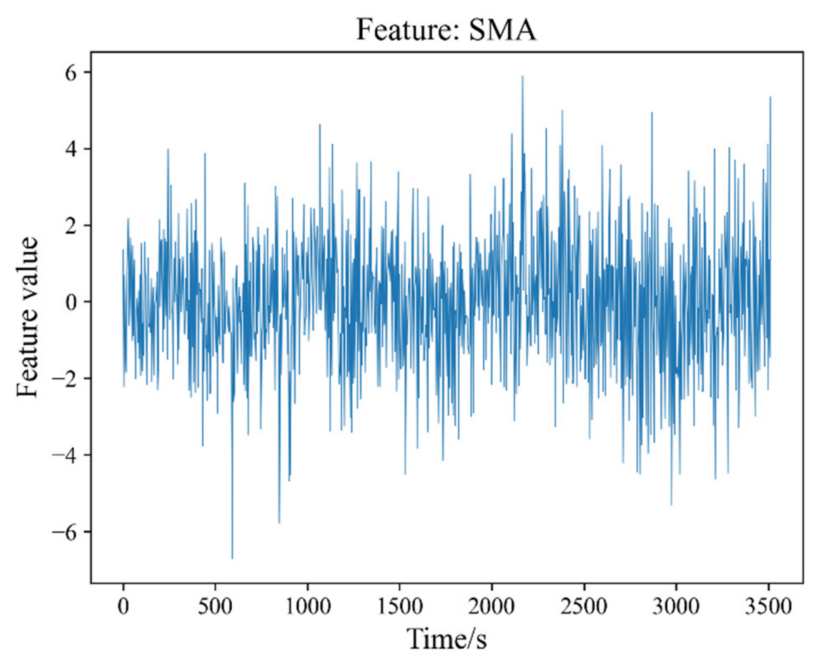

(h)

Figure 8. The mean values are calculated from the EMG features. The figures are plotted separately for each of the features: (a) MAV, (b) ZC, (c) WL, (d) SSC, (e) RMS, (f) MDF, (g) MPF, (h) SMA.

Table 1. The F values for the MANOV of the features.

\begin{tabular}{cccccccccc}
\hline Statistic & Factor & MAV & ZC & WL & SSC & RMS & MDF & MPF & SMA \\
\hline \multirow{2}{*}{ Mean } & Fatigue & 20.79 & 27.70 & 17.95 & 17.59 & 20.05 & 20.65 & 26.84 & 1.57 \\
& Individuals & 3772 & 3724 & 1810 & 4849 & 3463 & 4039 & 4008 & 0.02 \\
\hline \multirow{2}{*}{ Variance } & Fatigue & 10.67 & 9.26 & 20.17 & 10.08 & 10.90 & 20.01 & 27.02 & 28.87 \\
& Individuals & 391.3 & 349.9 & 398.3 & 534.9 & 382.9 & 610.1 & 450.1 & 1038.04 \\
\hline
\end{tabular}

\section{Discussion}

Muscle fatigue induced by long walking time changes the characteristics of sEMG signals. In this way, muscle fatigue affects the performances of the gait phase classifiers, potentially harming lower-limb wearable robots' ability to provide effective assistance. The practical applications of the gait phase classifiers in wearable robots demand a deeper understanding of the relationship between muscle fatigue and the classifiers' performance. Our study mainly performs the following three aspects of work: (1) A quantitative investigation on the impact of muscle fatigue on the performance of the classifiers using different 
features; (2) a training strategy that improves the robustness of the classifiers against the fatigue; (3) a statistical analysis to interpret the phenomena of the classification. These works can provide a reference for other studies, such as designing the robust human-machine interface and investigating muscle fatigue-induced sEMG changes.

Our results indicate that muscle fatigue has significant effects on the classifiers that use the features, including MAV, RMS, ZC, SSC, MDF, and MPF. Moreover, for the different features, the effects are not the same. For the single feature, SMA and WL may be the best choices if considering the robustness against fatigue. ZC and SSC present larger errors in our study both on the generalization to muscle fatigue and the accuracy of the classification. One of the possible reasons for still including them in the feature set is that they can improve the performance of the model when combined with other features.

The effects of muscle fatigue on the accuracies of the classifiers seem to be different from the phenomenon reported by the study [21]. They have performed a similar study that also used the MAV, ZC, WL, and SSC to train the classifiers by nonfatigue data, while testing them by fatigue data. Their results presented that muscle fatigue did not significantly affect the classifiers' accuracies. The reason for this divergence may be the different ways of inducing fatigue. In their study, they used the 90-s isometric contractions to induce short-term fatigue, while in our study, we investigate the effects of long-term fatigue. We choose long-term fatigue to conduct our study, since long-term fatigue seems to be more common fatigue during the usage of the lower-limb wearable robots.

By the practical test, we validate our proposed training strategy. This strategy is adding some of the fatigue data into the training set, which is pretty simple, but really works. As mentioned above, we add the first half of the fatigue data into the training set and use the second half data as the test set. The performance improvement may indicate that the subjects reach a stable state of fatigue after long-term walking. In this way, the data distribution might not change in the second half data of the third session. Therefore, the fatigue data adding into the training set can improve the performance of the classifiers under fatigue.

Through the analysis, we preliminarily explain the phenomena of classifier degradation in this study. The analysis results show the significant effects of muscle fatigue on the mean values and variances of all features, which meets the phenomena presented by several studies $[37,44-46]$. The difference of the statistical characteristics implies that the distributions of the features extracted from sEMG signals vary with the fatigue levels. That is to say, muscle fatigue is an important factor that causes diversities among the data sets, which may explain the classifier degradation when training the classifier with the nonfatigue data and testing it with the fatigue data. The generalization ability would be improved if the classifiers receive the more differentially distributed data during the training, which explains the effectiveness of our proposed training strategy.

Our training strategy has a practical value in the sEMG-based device control. In the traditional training of classifiers used in wearable robots, the training data is generally collected from a short-time experiment, which means that muscle fatigue has not yet been developed. The classifiers might deteriorate after a long time along with the fatigue deepening. Inspired by our study, when the subjects go into a state of fatigue after a long period of movement, the classifiers should be trained afresh by the data set that is composed of the nonfatigue data and the fatigue data. In this way, the gait phase classifiers may perform well both in the nonfatigue and fatigue states. For example, the study reported by [47] proposed a method to classify hand movements through the single-channel sEMG, which did not consider the fatigue situation, and the method can be improved by our strategy. Previous studies have proposed the methods of designing classifiers that are robust against fatigue, such as fuzzy neural networks and adaptive hybrid classifiers $[23,24]$. However, these methods may be accompanied by increasing the computation and the complexity of classifiers, while our strategy may be easier to use.

There are also some limitations in this study. First, we only test the classifiers using single features, and what kind of feature combinations can get better robustness against 
muscle fatigue is still unclear. Second, as mentioned above, the fatigue in our experiment is induced by long-time walking. Therefore, this paper only makes a rough division to the fatigue, and does not put forward an exact scale. Moreover, the practical validation of the proposed strategy on real devices controlled by sEMG, such as wearable robots or prostheses, needs to be implemented.

In future work, a multi-feature classifier should be tested by our method to find the feature set that has better stability under the interference of muscle fatigue. Our strategy will be performed on sEMG-controlled exoskeletons.

\section{Conclusions}

This study investigated the influence of muscle fatigue on the sEMG-based gait phase classification and found a solution to the performance deterioration of the classifier. We train the classifiers by the non-fatigue data and test them by the fatigue data. Our result shows that muscle fatigue has significant influences on gait phase classifiers, and reveals how muscle fatigue affects the classifiers using different features. By testing on the collected data, our training strategy that adds some fatigue data into the training set is effective. In order to explain the results of the classification, we perform the MANOVA on the features, which suggests that there are significant correlations between the features and muscle fatigue. The study contributes to our understanding of muscle fatigue in the domain of sEMG signals. Our findings can reference other studies in which muscle fatigue makes an impact, and our training strategy can be applied in sEMG-controlled devices to improve their performance under fatigue. However, this study is limited by the absence of an accurate scale of muscle fatigue, and we roughly divide the data into the fatigue set and the non-fatigue set. There still are several questions, such as the influence of muscle fatigue on the multi-feature classifiers, the validation of our training strategy on real devices, and the investigation of fatigue induced in different manners. These questions need to be answered by future work.

Author Contributions: Conceptualization, J.Z. and C.Y. (Chunzhi Yi); methodology, C.Y. (Chunzhi Yi); software, B.W.; validation, C.Y. (Chifu Yang), Z.D. and F.J.; formal analysis, J.Z.; investigation, C.Y. (Chifu Yang); resources, Z.D.; data curation, B.W.; writing-original draft preparation, J.Z.; writingreview and editing, C.Y. (Chunzhi Yi); visualization, Z.D.; supervision, F.J. All authors have read and agreed to the published version of the manuscript.

Funding: The work of this paper is funded by the project of National Key Research and Development Program of China (No. 2018YFC0806802).

Institutional Review Board Statement: The study was conducted according to the guidelines of the Declaration of Helsinki, and approved by the Ethics Committee of Chinese Ethics Committee of Registering Clinical Trials (ChiECRCT20200319).

Informed Consent Statement: Informed consent was obtained from all subjects involved in the study.

Conflicts of Interest: The authors declare no conflict of interest.

\section{References}

1. Bao, G.J.; Pan, L.F.; Fang, H.; Wu, X.Y.; Yu, H.Y.; Cai, S.B.; Yu, B.Q.; Wan, Y.H. Academic Review and Perspectives on Robotic Exoskeletons. IEEE Trans. Neural Syst. Rehabil. Eng. 2019, 27, 2294-2304. [CrossRef] [PubMed]

2. Rodriguez-Fernandez, A.; Lobo-Prat, J.; Font-Llagunes, J.M. Systematic review on wearable lower-limb exoskeletons for gait training in neuromuscular impairments. J. Neuroeng. Rehabil. 2021, 18, 1-21. [CrossRef] [PubMed]

3. Shi, D.; Zhang, W.X.; Zhang, W.; Ding, X.L. A Review on Lower Limb Rehabilitation Exoskeleton Robots. Chin. J. Mech. Eng. 2019, 32, 1-11. [CrossRef]

4. Gui, K.; Liu, H.H.; Zhang, D.G. A Practical and Adaptive Method to Achieve EMG-Based Torque Estimation for a Robotic Exoskeleton. IEEE-ASME Trans. Mechatron. 2019, 24, 483-494. [CrossRef]

5. Hussain, S.; Jamwal, P.K.; Van Vliet, P.; Ghayesh, M.H. State-of-the-Art Robotic Devices for Wrist Rehabilitation: Design and Control Aspects. IEEE Trans. Hum. Mach. Syst. 2020, 50, 361-372. [CrossRef]

6. Lyu, M.X.; Chen, W.H.; Ding, X.L.; Wang, J.H.; Pei, Z.C.; Zhang, B.C. Development of an EMG-Controlled Knee Exoskeleton to Assist Home Rehabilitation in a Game Context. Front. Neurorobot. 2019, 13, 67. [CrossRef] 
7. Sawicki, G.S.; Beck, O.N.; Kang, I.; Young, A.J. The exoskeleton expansion: Improving walking and running economy. J. Neuroeng. Rehabil. 2020, 17, 1-9. [CrossRef]

8. Chaparro-Rico, B.D.M.; Cafolla, D.; Tortola, P.; Galardi, G. Assessing Stiffness, Joint Torque and ROM for Paretic and Non-Paretic Lower Limbs during the Subacute Phase of Stroke Using Lokomat Tools. Appl. Sci. 2020, 10, 6168. [CrossRef]

9. Kantoch, E. Recognition of Sedentary Behavior by Machine Learning Analysis of Wearable Sensors during Activities of Daily Living for Telemedical Assessment of Cardiovascular Risk. Sensors 2018, 18, 3219. [CrossRef]

10. Hargrove, L.J.; Scheme, E.J.; Englehart, K.B.; Hudgins, B.S. Multiple Binary Classifications via Linear Discriminant Analysis for Improved Controllability of a Powered Prosthesis. IEEE Trans. Neural Syst. Rehabil. Eng. 2010, 18, 49-57. [CrossRef]

11. Kim, J.S.; Jeong, H.; Son, W.; IEEE. A new means of HCI: EMG-mouse. In Proceedings of the 2004 IEEE International Conference on Systems, Man \& Cybernetics, The Hague, The Netherlands, 10-13 October 2004; Volume 1-7, pp. 100-104.

12. Scheme, E.J.; Englehart, K.B.; Hudgins, B.S. Selective Classification for Improved Robustness of Myoelectric Control under Nonideal Conditions. IEEE Trans. Biomed. Eng. 2011, 58, 1698-1705. [CrossRef]

13. Kyranou, I.; Vijayakumar, S.; Erden, M.S. Causes of Performance Degradation in Non-invasive Electromyographic Pattern Recognition in Upper Limb Prostheses. Front. Neurorobot. 2018, 12, 58. [CrossRef]

14. Naeije, M.; Zorn, H. Changes in the power spectrum of the surface electromyogram of the human masseter muscle due to local muscular fatigue. Arch. Oral Biol. 1981, 26, 409-412. [CrossRef]

15. Zhang, G.; Morin, E.; Zhang, Y.; Etemad, S.A. Non-invasive detection of low-level muscle fatigue using surface EMG with wavelet decomposition. In Proceedings of the Annual International Conference of the IEEE Engineering in Medicine and Biology Society, Honolulu, HI, USA, 18-21 July 2018; pp. 5648-5651. [CrossRef]

16. Camata, T.V.; Lacerda, T.R.; Altimari, L.R.; Bortolloti, H.; Fontes, E.B.; Dantas, J.L.; Nakamura, F.Y.; Abrao, T.; Chacon-Mikahil, M.P.T.; Moraes, A.C. Association between the electromyographic fatigue threshold and ventilatory threshold. Electromyogr. Clin. Neurophysiol. 2009, 49, 305-310.

17. De Luca, C.J. The use of surface electromyography in biomechanics. J. Appl. Biomech. 1997, 13, 135-163. [CrossRef]

18. Thongpanja, S.; Phinyomark, A.; Phukpattaranont, P.; Limsakul, C. A Feasibility Study of Fatigue and Muscle Contraction Indices Based on EMG Time-dependent Spectral Analysis. In Iseec; Kaewkhao, J., Limsuwan, P., Yupapin, P.P., JanJai, S.J.J., Yupapin, P., Eds.; Elsevier: Amsterdam, The Netherlands, 2012; Volume 32, pp. 239-245.

19. Hussain, M.S.; Mamun, M. Effectiveness of the Wavelet Transform on the Surface EMG to Understand the Muscle Fatigue During Walk. Meas. Sci. Rev. 2012, 12, 28-33. [CrossRef]

20. Yochum, M.; Bakir, T.; Lepers, R.; Binczak, S. Estimation of Muscular Fatigue Under Electromyostimulation Using CWT. IEEE Trans. Biomed. Eng. 2012, 59, 3372-3378. [CrossRef]

21. Tkach, D.; Huang, H.; Kuiken, T.A. Study of stability of time-domain features for electromyographic pattern recognition. J. Neuroeng. Rehabil. 2010, 7, 21. [CrossRef]

22. Bukhari, W.M.; Yun, C.J.; Kassim, A.M.; Tokhi, M.O. Study of K-Nearest Neighbour Classification Performance on Fatigue and Non-Fatigue EMG Signal Features. Int. J. Adv. Comput. Sci. Appl. 2020, 11, 41-47. [CrossRef]

23. Song, J.-H.; Jung, J.-W.; Lee, S.-W.; Bien, Z. Robust EMG pattern recognition to muscular fatigue effect for powered wheelchair control. J. Intell. Fuzzy Syst. 2009, 20, 3-12. [CrossRef]

24. Ding, Q.; Zhao, X.; Han, J.; Bu, C.; Wu, C. Adaptive Hybrid Classifier for Myoelectric Pattern Recognition Against the Interferences of Outlier Motion, Muscle Fatigue, and Electrode Doffing. IEEE Trans. Neural Syst. Rehabil. Eng. 2019, 27, 1071-1080. [CrossRef]

25. Mainardi, E.; Urbano, E.; Davalli, A. Design of a new EMG sensor for upper limb prosthetic control and real time frequency analysis. In Proceedings of the 2008 MyoElectric Controls/Powered Prosthetics Symposium, Fredericton, NB, Canada, 13-15 August 2008.

26. Granacher, U.; Wolf, I.; Wehrle, A.; Bridenbaugh, S.; Kressig, R.W. Effects of muscle fatigue on gait characteristics under single and dual-task conditions in young and older adults. J. Neuroeng. Rehabil. 2010, 7, 56. [CrossRef]

27. Barbieri, F.A.; Rocha dos Santos, P.C.; Simieli, L.; Orcioli-Silva, D.; van Dieen, J.H.; Bucken Gobbi, L.T. Interactions of age and leg muscle fatigue on unobstructed walking and obstacle crossing. Gait Posture 2014, 39, 985-990. [CrossRef]

28. Morrison, S.; Colberg, S.R.; Parson, H.K.; Neumann, S.; Handel, R.; Vinik, E.J.; Paulson, J.; Vinik, A.I. Walking-Induced Fatigue Leads to Increased Falls Risk in Older Adults. J. Am. Med. Dir. Assoc. 2016, 17, 402-409. [CrossRef]

29. Crozara, L.F.; Castro, A.; De Almeida Neto, A.F.; Laroche, D.P.; Cardozo, A.C.; Goncalves, M. Utility of electromyographic fatigue threshold during treadmill running. Muscle Nerve 2015, 52, 1030-1039. [CrossRef]

30. Hanon, C.; Thepaut-Mathieu, C.; Vandewalle, H. Determination of muscular fatigue in elite runners. Eur. J. Appl. Physiol. 2005, 94, 118-125. [CrossRef]

31. Yu, B.; Queen, R.M.; Abbey, A.N.; Liu, Y.; Moorman, C.T.; Garrett, W.E. Hamstring muscle kinematics and activation during overground sprinting. J. Biomech. 2008, 41, 3121-3126. [CrossRef]

32. Chowdhury, R.H.; Reaz, M.B.I.; Ali, M.A.M. Determination of Muscle Fatigue in SEMG Signal Using Empirical Mode Decomposition. In Proceedings of the IEEE International Conference on Biomedical Engineering and Sciences, Miri, Malaysia, 8-10 December 2014; pp. 932-937.

33. Knaflitz, M.; Molinari, F. Assessment of muscle fatigue during biking. IEEE Trans. Neural Syst. Rehabil. Eng. 2003, 11, 17-23. [CrossRef] [PubMed] 
34. Singh, V.P.; Kumar, D.K.; Polus, B.; Fraser, S. Strategies to identify changes in SEMG due to muscle fatigue during cycling. J. Med. Eng. Technol. 2007, 31, 144-151. [CrossRef] [PubMed]

35. Tokuyasu, T.; Kushizaki, S.; Matsumoto, S.; Kitawaki, T. Development of automatic positioning system for bicycle saddle based on lower limb's EMG signals during pedaling motion. In Proceedings of the 2013 IEEE 6th International Workshop on Computational Intelligence and Applications (IWCIA), Hiroshima, Japan, 13 July 2013; pp. 27-32.

36. Rocha, V.d.A., Jr.; do Carmo, J.C.; Nascimento, F.A.d.O. Weighted-Cumulated S-EMG Muscle Fatigue Estimator. IEEE J. Biomed. Health Inform. 2018, 22, 1854-1862. [CrossRef] [PubMed]

37. De Luca, C.J. Use of the surface emg signal for performance evaluation of back muscles. Muscle Nerve 1993, 16, 210-216. [CrossRef]

38. Gonzalez-Izal, M.; Malanda, A.; Navarro-Amezqueta, I.; Gorostiaga, E.M.; Mallor, F.; Ibanez, J.; Izquierdo, M. EMG spectral indices and muscle power fatigue during dynamic contractions. J. Electromyogr. Kinesiol. 2010, 20, 233-240. [CrossRef]

39. Phinyomark, A.; Campbell, E.; Scheme, E. Surface electromyography (EMG) signal processing, classification, and practical considerations. In Biomedical Signal Processing; Springer: Berlin/Heidelberg, Germany, 2020; pp. 3-29.

40. Meng, M.; Luo, Z.; She, Q.; Ma, Y. Automatic recognition of gait mode from EMG signals of lower limb. In Proceedings of the 2010 the 2nd International Conference on Industrial Mechatronics and Automation, Wuhan, China, 30-31 May 2010; pp. 282-285.

41. Phinyomark, A.; Phukpattaranont, P.; Limsakul, C. Feature reduction and selection for EMG signal classification. Expert Syst. Appl. 2012, 39, 7420-7431. [CrossRef]

42. Lorrain, T.; Jiang, N.; Farina, D. Influence of the training set on the accuracy of surface EMG classification in dynamic contractions for the control of multifunction prostheses. J. Neuroeng. Rehabil. 2011, 8, 25. [CrossRef]

43. Gokgoz, E.; Subasi, A. Comparison of decision tree algorithms for EMG signal classification using DWT. Biomed. Signal Process. Control 2015, 18, 138-144. [CrossRef]

44. Cifrek, M.; Medved, V.; Tonkovic, S.; Ostojic, S. Surface EMG based muscle fatigue evaluation in biomechanics. Clin. Biomech. 2009, 24, 327-340. [CrossRef]

45. Katsis, C.D.; Ntouvas, N.E.; Bafas, C.G.; Fotiadis, D.I. Assessment of Muscle Fatigue during Driving Using Surface EMG. In Proceedings of the 2nd IASTED International Conference on Biomedical Engineering, Innsbruck, Austria, 16-18 February 2004; pp. 259-262.

46. McDonald, A.C.; Mulla, D.M.; Keir, P.J. Using EMG Amplitude and Frequency to Calculate a Multimuscle Fatigue Score and Evaluate Global Shoulder Fatigue. Hum. Factors 2019, 61, 526-536. [CrossRef]

47. Arozi, M.; Caesarendra, W.; Ariyanto, M.; Munadi, M.; Setiawan, J.D.; Glowacz, A. Pattern Recognition of Single-Channel sEMG Signal Using PCA and ANN Method to Classify Nine Hand Movements. Symmetry 2020, 12, 541. [CrossRef] 